\title{
MITTEILUNGEN
}

\section{Konsolidierter Parlamentarismus? Lettland und Ungarn 20 Jahre nach der Wende. Eine Veranstaltung der DVParl am 13. Mai 2009 in Berlin}

Transformationsstaaten erwecken immer wieder das Interesse des Betrachters, weil sie um die Sicherung ihres Bestehens ringen. Während in etablierten Demokratien von einer dauerhaften Stabilität der politischen Ordnung ausgegangen werden kann, sind junge Demokratien nicht davor gefeit, ausgehöhlt zu werden oder gar zusammenzubrechen. Politische Institutionen können dazu beitragen, dass demokratische Neulinge nicht bloß als formale Demokratien bestehen, sondern derart in ihrem Bestand gefestigt werden, dass sie auch gegen größere Krisen gewappnet sind.

Am 13. Mai 2009 hat die Deutsche Vereinigung für Parlamentsfragen Lettland und Ungarn in den Blick genommen: Wie konsolidiert ist deren Parlamentarismus zwanzig Jahre nach dem Systemwechsel? ${ }^{1}$ Eingeladen waren Zoltán Balog (ungarischer Abgeordneter der „Bürgerlichen Partei Fidesz") und Martin Virsis (Generalinspektor im lettischen Außenministerium). Heinrich Oberreuter (Universität Passau, Akademie für Politische Bildung Tutzing) moderierte die Veranstaltung.

Beide Länder verfügen - wenn man die Vorläufer der Zwischenkriegszeit zunächst außer Acht lässt - erst seit zwanzig Jahren über ein durch Verfassung und Wahlen demokratisch legitimiertes Parlament. Am 5. und 6. Juni 1993 wählten die Letten zum ersten Mal in ihrer neuerlichen Unabhängigkeit, die sie seit dem Frühjahr 1990 in aller Öffentlichkeit beanspruchten, aber erst im Sommer 1993 erhielten, ein freies Parlament. Die Ungarn taten dies bereits in zwei Wahlgängen am 25. März und 8. April 1990. Beide Staaten haben gemein, dass der neue Parlamentarismus nicht auf einer völlig neuen Verfassung fußt. Zoltán Balog berichtete, dass in Ungarn formal noch immer die stalinistische Verfassung der ehemaligen Volksrepublik gelte. Nach jedoch zwanzig Verfassungsänderungen seit 1989 seien lediglich zwei Dinge konstant geblieben: die Jahreszahl 1949 für das Inkrafttreten und die Festlegung, dass die Hauptstadt Budapest ist. Nach der Darstellung von Martin Virsis wur-

1 Vgl. Georg Paul Hefty, Konsolidierter Parlamentarismus? Gemessen an dem in Deutschland Gewohnten: Eigentümlichkeiten in Lettland und Ungarn, in: FAZ vom 6. Juni 2009, S. 10. Zur Dokumentation der Parlamentsentwicklungen seit der politischen Wende in den beiden Staaten siehe für Ungarn: Jürgen Dieringer, Zwischen Parlamentsvorbehalt und Regierungsdominanz: die wachsende Bedeutung des ungarischen Parlaments im europäischen Integrationsprozess, in: ZParl, 38. Jg. (2007), H. 4, S. 764 - 775; Andrea Gawrich, Die jüngsten Parlamentswahlen in Polen, Ungarn und Tschechien, in: ZParl, 34. Jg. (2003), H. 2, S. 270 - 284; Jürgen Dieringer, Die ungarischen Parlamentswahlen 1998, in: ZParl, 29. Jg. (1998), H. 4, S. 648 - 660. Vgl. für Lettland: Axel Reetz, Die vierten Parlamente in Estland, Lettland und Litauen: Ähnliche Voraussetzungen, verschiedene Pfade, in: ZParl, 36. Jg. (2005), H. 2, S. 326 - 348; ders., Die dritten Parlamente der Esten, Letten und Litauer, in: ZParl, 33. Jg. (2002), H. 2, S. 290 - 305; ders., Demokratische Transformation im Baltikum, in: ZParl, 30. Jg. (1999), H. 4, S. 924 - 955; ders., Wahlen im Baltikum seit 1990. Parlamente in den Paradoxien der Selbstbestimmung, in: Winfried Steffani / Uwe Thaysen (Hrsg.), Demokratie in Europa: Zur Rolle der Parlamente (Sonderband zum 25jährigen Bestehen der Zeitschrift für Parlamentsfragen), Opladen 1995, S. 300 323. 
de in Lettland die Verfassung von 1923 wieder in Kraft gesetzt, um die rechtliche Identität mit der ersten Republik zum Ausdruck zu bringen. Hinzugefügt wurde lediglich ein Grundrechtsteil. Wie ist jedoch der Parlamentarismus in den beiden Ländern ausgestaltet? In welcher Form werden die primären Funktionen Wahl, Kontrolle, Gesetzgebung und Repräsentation durch die Parlamente erfüllt? ${ }^{2}$ Betrachtet man diese weniger als genuine Parlamentsfunktionen, sondern vielmehr als komplexe Prozesse eines Gewaltenteilungssystems staatlicher Institutionen, eröffnet ein solcher Blick auf das Parlament Einblicke in die Funktionslogik des gesamten politischen Systems. ${ }^{3}$

Im unikameral organisierten parlamentarischen Regierungssystem Ungarns steht die Nationalversammlung laut Verfassung als einziges direkt gewähltes Organ im Zentrum des politischen Entscheidungsgefüges. Die Anzahl von 386 Abgeordneten ist im Verhältnis zur Bevölkerung des Landes groß. Minderheiten sind nicht fest im Parlament vertreten, auch wenn, wie Balog bemerkte, die Verfassung den Anspruch einer Parlamentsvertretung impliziert. Wahlfunktionen bestehen gegenüber dem Staatspräsidenten und dem Ministerpräsidenten, nicht aber gegenüber den Ministern. Balog beschrieb, wie das konstruktive Misstrauensvotum zweimal gebraucht - nach der Meinung des Staatspräsidenten missbraucht - wurde, indem sich keine neue, sondern die alte Parlamentsmehrheit hinter einem neuen Kandidaten vereinte. Die Kontrollfunktion der Abgeordneten wird mit Hilfe von Interpellationsrechten und Untersuchungsausschüssen ausgeübt. Ein weiteres Instrument sind die seit 1995 eingesetzten Ombudsmänner. Gesetze können vom Staatspräsidenten, der Regierung, einem Ausschuss oder einem Abgeordneten eingebracht werden. Das Vetorecht des Staatspräsidenten ist suspensiv und kann mit einfacher Mehrheit überstimmt werden. Die formal starke Stellung des Parlaments wird jedoch begrenzt durch die de facto gesteigerte Bedeutung des Regierungschefs in der ungarischen „Kanzlerdemokratie“. Balog unterstrich, dass diese Entwicklung auch in der Verdoppelung der Zahl der Beschäftigten im Kanzleramt abzulesen sei.

Gestärkt wird die Legislative gegenüber der Exekutive hingegen durch die große Zahl an so genannten Zweidrittelgesetzen. In nunmehr zwölf Gesetzgebungsfeldern besteht dieses hohe Mehrheitserfordernis. Es wurde eingeführt, um die Regierungsmehrheit in ihren Möglichkeiten zu begrenzen und die polarisierten Parteien in der Wendezeit zu einem Konsens zu bewegen. Zwanzig Jahre nach der Ausrufung der Republik gelingt es den Akteuren jedoch immer seltener, einen Minimalkonsens herzustellen, was laut Balog Reformen stark hemmt und zu einer parlamentarischen Dauerblockade führt.

Die lettische Verfassung sieht ein parlamentarisches Regierungssystem mit einem vom Parlament gewählten Staatspräsidenten vor. Das wichtigste Staatsorgan ist das aus einer Kammer bestehende Parlament mit 100 Abgeordneten, die Saeima. Der Staatspräsident hat eingeschränkte Möglichkeiten, den politischen Prozess zu beeinflussen: So kann er ein Gesetz zur erneuten Durchsicht an das Parlament rücküberweisen oder die Verkündung eines

2 Seit Walter Bagehot werden die Leistungen von Parlamenten in Funktionskatalogen zusammengestellt; ders., The English Constitution, London 1867. Eine Zusammenstellung verschiedener Kataloge findet sich bei Peter Schindler, Datenhandbuch zur Geschichte des Deutschen Bundestages 1949 bis 1999, Bd. II, Baden-Baden 1999, S. 2834 ff.

3 Vgl. Roland Lhotta, Parlamentarismus als Gewaltenteilung: Institutionelle Ausprägungen komplexer Demokratie am Beispiel von Repräsentation, Regierungsbildung und Gesetzgebung, in: Helmar Schöne / Julia von Blumenthal (Hrsg.), Parlamentarismusforschung in Deutschland, BadenBaden 2009, S. 259 - 278. 
Gesetzes für zwei Monate aussetzen. Über ein eingeschränkt suspensives Veto verfügt auch das Parlament auf Antrag eines Drittels seiner Mitglieder. Eine Volksabstimmung entscheidet dann über die Annahme des Gesetzes. Ein Plebiszit ist ebenso notwendig, wenn der Staatspräsident das Parlament aufösen möchte. Erst wenn die Mehrheit der Wähler sich für diesen Antrag ausspricht, kann die Volksvertretung aufgelöst werden. Virsis wies auf die Besonderheit hin, dass sich der Präsident, sollte dieses Referendum scheitern, selbst neu zur Wahl stellen muss.

Im Vergleich zu Ungarn verfügt der lettische Ministerpräsident über geringere Befugnisse. Virsis sieht ihn als primus inter pares; Ministerpräsident und Regierung sind ausschließlich dem Parlament verantwortlich. Da es der Saeima möglich ist, durch ein Misstrauensvotum den Regierungschef sowie einzelne Minister zum Rücktritt zu zwingen, kann sich nur schwer eine Richtlinienkompetenz des Ministerpräsidenten entwickeln, nicht einmal eine Vormachtstellung im Kabinett, weil dessen einzelne Mitglieder stets abhängig vom Parlament bleiben.

Ein Hauptcharakteristikum des politischen Systems in Lettland stellt, laut Martin Virsis, die stark zersplitterte politische Landschaft dar, die die Regierungsbildung erschwert. Das Vielparteiensystem ist instabil und geprägt von Spaltungen, Vereinigungen, Umbenennungen und Neugründungen. Virsis erklärte, dass in den neunziger Jahren die meisten Parteien klein und organisatorisch schwach waren, bei manchen handelte es sich mehr um Personenverbände als um politische Parteien im westeuropäischen Sinne. Auch das Anheben der Sperrklausel von vier auf fünf Prozent bei der Parlamentswahl 1995 führte nicht zu einem Mehr an Stabilität. Bei der letzten Wahl 2006 schafften sieben Parteien den Sprung in die Saeima. Zwar wurde die regierende Mitte-Rechts-Koalition im Amt bestätigt; sie musste ihr Bündnis jedoch um einen vierten Partner erweitern. Die für parlamentarische Regierungssysteme notwendige funktionelle Einheit zwischen Regierung und Parlamentsmehrheit ist in Lettland vorhanden, aber wenig stabil. Für die bisherigen Regierungen war es aufgrund der knappen Mehrheitsverhältnisse im Parlament meist kompliziert, eine verlässliche parlamentarische Mehrheit zu gewinnen und dauerhaft aufrecht zu erhalten.

In der anschließenden Diskussion wurden aktuelle politische und wirtschaftliche Themen angesprochen. So fragte Nils Diederich (Freie Universität Berlin), inwiefern die politischen Systeme in den beiden Ländern in der Lage sind, die Auswirkungen der Wirtschaftsund Finanzkrise zu meistern. Einerseits ist vorstellbar, dass schwache Institutionen nur begrenzt reagieren können, andererseits könnte allerdings das Gegenteil eintreten, dass gerade in einer solchen Notsituation die institutionellen Hemmnisse transzendiert werden.

Zur Beantwortung der Frage führte Balog grundlegend an, dass in Ungarn weniger die Schwäche des Parlamentes als vielmehr die der Regierung die Handlungsfähigkeit einschränke. Die Wirtschaftskrise kam nur zur bestehenden politischen Krise hinzu, so dass es noch schwieriger wurde, Konsens für Reformen zu erzielen. Nachdem Ministerpräsident Ferenc Gyurcsany zugegeben hatte, die Bevölkerung vor der Parlamentswahl 2006 belogen zu haben, gelang es trotz bestehender Mehrheit im Parlament zwischen Oktober 2008 und Mitte Mai 2009 nicht, auch nur ein einziges Gesetz zur Finanzkrise zu verabschieden. Virsis' Einschätzung nach ist in Lettland das Parlament das Problem und gleichzeitig die Lösung in Krisenzeiten. Ohne die Saeima kann kein Finanzpaket auf den Weg gebracht werden. Es besteht die Hoffnung, dass die Wirtschafts- und Finanzkrise ein einendes Ziel für die zerstrittenen Fraktionen darstellt und damit, wie nach dem lettischen Beitritt zur Europäischen Union und zur NATO, die Parteien nicht weiter auseinanderdriften. 
Zusammenfassend lässt sich sagen, dass der Systemwechsel in beiden Staaten durch eine dreifache Gleichzeitigkeit bestimmt wurde: Neben dem Übergang von einem nicht-demokratischen Staat zur Demokratie und von der Planwirtschaft zur Marktwirtschaft mussten leistungsfähige staatliche Strukturen geschaffen werden. Der Abend zeigte, dass die DVParl dem Thema Konsolidierung zu Recht ein Fragezeichen hinzugefügt hat. Institutionelle Schranken und ein fehlender Minimalkonsens der politischen Lager führen in Ungarn zu einer parlamentarischen Blockade und messbarer Unzufriedenheit in der Bevölkerung. In Lettland erschwert die Zersplitterung der Parteienlandschaft die Bildung von Parlamentsmehrheiten und beeinträchtigt damit die Stabilität der Regierung - hinzu kommt der Wettstreit um Zuständigkeiten zwischen Parlament und Staatspräsidenten. Dies lässt nur bedingt einen konsolidierten Parlamentarismus in Lettland und Ungarn erkennen. Der Blick in die Transformationstheorie zeigt, dass Diskussionen zum Stand der Konsolidierung neben der Analyse des Parlamentarismus - fünf sich wechselseitig beeinflussende Arenen einbeziehen sollten: ein ausdifferenziertes System politischer Institutionen, die Etablierung rechtsstaatlicher Verhältnisse, eine funktionsfähige Bürokratie, eine „economic society“, die zwischen Staat und Markt vermittelt, sowie eine lebhafte freie Zivilgesellschaft. ${ }^{4}$

Alexander Kühne

4 Vgl. Juan J. Linz / Alfred C. Stepan, Problems of Democratic Transition and Consolidation. Southern Europe, South America, and Post-Communist Europe, Baltimore / London 1996. 